Article

\title{
Applications of Lyapunov Functions to Caputo Fractional Differential Equations
}

\author{
Ravi Agarwal ${ }^{1,2}$, Snezhana Hristova ${ }^{3, *}$ and Donal O'Regan ${ }^{4}$ \\ 1 Department of Mathematics, Texas A\&M University-Kingsville, Kingsville, TX 78363, USA; \\ agarwal@tamuk.edu \\ 2 Distinguished University Professor of Mathematics, Florida Institute of Technology, Melbourne, \\ FL 32901, USA \\ 3 Department of Applied Mathematics and Modeling, University of Plovdiv, Tzar Asen 24, \\ 4000 Plovdiv, Bulgaria \\ 4 School of Mathematics, Statistics and Applied Mathematics, National University of Ireland, \\ H91 CF50 Galway, Ireland; donal.oregan@nuigalway.ie \\ * Correspondence: snehri@gmail.com
}

Received: 28 September 2018; Accepted: 24 October 2018; Published: 30 October 2018

check for updates

\begin{abstract}
One approach to study various stability properties of solutions of nonlinear Caputo fractional differential equations is based on using Lyapunov like functions. A basic question which arises is the definition of the derivative of the Lyapunov like function along the given fractional equation. In this paper, several definitions known in the literature for the derivative of Lyapunov functions among Caputo fractional differential equations are given. Applications and properties are discussed. Several sufficient conditions for stability, uniform stability and asymptotic stability with respect to part of the variables are established. Several examples are given to illustrate the theory.
\end{abstract}

Keywords: stability; Caputo derivative; Lyapunov functions; fractional differential equations

AMS Subject Classifications: 34A34; 34A08; 34D20

\section{Introduction}

Recently, fractional calculus has attracted much attention since it plays an important role in many fields of science and engineering since the behavior of many systems, such as physical phenomena having memory and genetic characteristics, can be adequately modeled by fractional differential systems (see, for example, [1-3]). The question of stability is of interest in physical and biological systems, such as the fractional Duffing oscillator [4], fractional predator-prey and rabies models [5], etc. and stability theory of fractional differential equations(FDEs) is widely applied to chaos and chaos synchronization [6] because of its potential applications in control processing and secure communication. We refer to the book [7] about several applications in Physics, the review paper [8] about applications to Engineering and the book [9] about applications to Financial Economics. The analysis on stability of fractional differential equations is more complex than classical differential equations, since fractional derivatives are nonlocal and have weakly singular kernels. Several results were obtained such as structural stability of systems with Riemann-Liouville derivative [10]), continuous dependence of solution on initial conditions for Caputo nonautonomous fractional differential equations [11], stability in the sense of Lyapunov by using Gronwall's inequality and Schwartz's inequality [12], Mittag-Leffler stability [13], and local asymptotic stability [14]. Basic results on stability of fractional differential equations including linear, nonlinear, as well as with time-delay are given in [15]. There are several approaches in the literature to study stability, one of which is 
the Lyapunov approach. This approach is successfully applied to study various types of stability for different kinds of differential equations (see, for example, [16-19]. As is mentioned in [20], there are several difficulties encountered when one applies the Lyapunov technique to fractional differential equations. Results on stability in the literature via Lyapunov functions could be divided into two main groups:

- continuously differentiable Lyapunov functions (see, for example, the papers [13,21-23]. Different types of stability are discussed using the Caputo derivative of Lyapunov functions which depends significantly of the unknown solution of the fractional equation.

- continuous Lyapunov functions (see, for example, the papers $[24,25]$ in which the authors use the derivative of a Lyapunov function which is similar to the Dini derivative of Lyapunov functions.

The stability theory presented here was initially developed in a series of papers [16]. The purpose of this paper is to refine the fundamental theorems and to discuss and illustrate some of these results and to present some new ones. A Caputo fractional Dini derivative of a Lyapunov function among nonlinear Caputo fractional differential equations is presented. This type of derivative was introduced in [16] and used to study stability and asymptotic stability [16] of Caputo fractional differential equations, and for stability of Caputo fractional differential equations with non-instantaneous impulses [17]. Comparison results using this definition and scalar fractional differential equations are presented and several sufficient conditions for stability, uniform stability, asymptotic stability with respect to part of the variables are given. Most of the results are illustrated with examples.

\section{Notes on Fractional Calculus}

Fractional calculus generalizes the derivative and the integral of a function to a non-integer order [24,26-29]. In engineering, the fractional order $q$ is often less than 1 , so we restrict our attention to $q \in(0,1)$.

1: The Riemann-Liouville (RL) fractional derivative of order $q \in(0,1)$ of $m(t)$ is given by (see, for example, Section 1.4.1.1 [26], or [28]

$$
{ }_{t_{0}}^{R L} D_{t}^{q} m(t)=\frac{1}{\Gamma(1-q)} \frac{d}{d t} \int_{t_{0}}^{t}(t-s)^{-q} m(s) d s, \quad t \geq t_{0}
$$

where $\Gamma($.$) denotes the Gamma function.$

2: The Caputo fractional derivative of order $q \in(0,1)$ is defined by (see, for example, Section 1.4.1.3 [26]

$$
{ }_{t_{0}}^{c} D_{t}^{q} m(t)=\frac{1}{\Gamma(1-q)} \int_{t_{0}}^{t}(t-s)^{-q} m^{\prime}(s) d s, \quad t \geq t_{0} .
$$

The properties of the Caputo derivative are quite similar to those of ordinary derivatives. In addition, the initial conditions of fractional differential equations with the Caputo derivative has a clear physical meaning and as a result the Caputo derivative is usually used in real applications.

The relations between both types of fractional derivatives are given by ${ }_{t_{0}}^{c} D_{t}^{q} m(t)={ }_{t_{0}}^{R L} D_{t}^{q}\left[m(t)-m\left(t_{0}\right)\right]$ and ${ }_{t_{0}}^{c} D_{t}^{q} m(t)={ }_{t_{0}}^{R L} D_{t}^{q} m(t)$ iff $m\left(t_{0}\right)=0$.

3: The Grunwald-Letnikov fractional derivative is given by (see, for example, [26] (Section 1.4.1.2))

$$
{ }_{t_{0}}^{G L} D_{t}^{q} m(t)=\lim _{h \rightarrow 0} \frac{1}{h^{q}} \sum_{r=0}^{\left[\frac{t-t_{0}}{h}\right]}(-1)^{r}\left(\begin{array}{l}
q \\
r
\end{array}\right) m(t-r h), \quad t \geq t_{0},
$$


and the Grunwald-Letnikov fractional Dini derivative by

$$
{ }_{t_{0}}^{G L} D_{+}^{q} m(t)=\limsup _{h \rightarrow 0+} \frac{1}{h^{q}} \sum_{r=0}^{\left[\frac{t-t_{0}}{h}\right]}(-1)^{r}\left(\begin{array}{l}
q \\
r
\end{array}\right) m(t-r h), \quad t \geq t_{0}
$$

$\left[\frac{t-t_{0}}{h}\right]$ denotes the integer part of the fraction $\frac{t-t_{0}}{h}$.

Proposition 1 ([27] (Theorem 2.25)). Let $m \in C^{1}\left[t_{0}, b\right]$. Then, for $t \in\left(t_{0}, b\right],{ }_{t_{0}}^{G L} D_{t}^{q} m(t)={ }_{t_{0}}^{R L} D_{t}^{q} m(t)$.

In addition, according to [27] (Lemma 3.4),${ }_{t_{0}}^{c} D_{t}^{q} m(t)={ }_{t_{0}}^{R L} D_{t}^{q} m(t)-m\left(t_{0}\right) \frac{\left(t-t_{0}\right)-q}{\Gamma(1-q)}$ holds.

From the relation between the Caputo fractional derivative and the Grunwald-Letnikov fractional derivative using the above, we define the Caputo fractional Dini derivative as

$$
{ }_{t_{0}}^{c} D_{+}^{q} m(t)={ }_{t_{0}}^{G L} D_{+}^{q}\left[m(t)-m\left(t_{0}\right)\right]
$$

i.e.,

$$
{ }_{t_{0}}^{c} D_{+}^{q} m(t)=\limsup _{h \rightarrow 0+} \frac{1}{h^{q}}\left[m(t)-m\left(t_{0}\right)-\sum_{r=1}^{\left[\frac{t-t_{0}}{h}\right]}(-1)^{r+1}\left(\begin{array}{l}
q \\
r
\end{array}\right)\left(m(t-r h)-m\left(t_{0}\right)\right)\right] .
$$

Definition 1 (Ref. [30]). We say $m \in C^{q}\left(\left[t_{0}, T\right], \mathbb{R}^{n}\right)$ if $m(t)$ is differentiable (i.e., $m^{\prime}(t)$ exists), the Caputo derivative ${ }_{t_{0}}^{c} D_{t}^{q} m(t)$ exists and satisfies (1) for $t \in\left[t_{0}, T\right]$.

Remark 1. Definition 1 could be extended to any interval $I \subset \mathbb{R}_{+}$.

Remark 2. If $m \in C^{q}\left(\left[t_{0}, T\right], \mathbb{R}^{n}\right)$, then ${ }_{t_{0}}^{c} D_{+}^{q} m(t)={ }_{t_{0}}^{c} D_{t}^{q} m(t)$.

In this paper, we will use the following results:

Lemma 1 (Ref. [21]). Let $x \in C^{q}\left(\left[t_{0}, \infty\right), \mathbb{R}^{n}\right)$. Then, for any $t \geq t_{0}$, the inequality

$$
{ }_{t_{0}}^{c} D_{t}^{q}\left(x^{T}(t) x(t)\right) \leq 2 x^{T}(t){ }_{t_{0}}^{c} D_{t}^{q} x(t)
$$

holds.

\section{Statement of the Problem and Definitions for Stability}

Consider the initial value problem (IVP) for the system of fractional differential equations (FrDE) with a Caputo derivative for $0<q<1$,

$$
{ }_{t_{0}}^{c} D_{t}^{q} x(t)=f(t, x(t)), \quad x\left(t_{0}\right)=x_{0},
$$

where $x, x_{0} \in \mathbb{R}^{n}, f \in C\left[\mathbb{R}_{+} \times \mathbb{R}^{n}, \mathbb{R}^{n}\right], f(t, 0) \equiv 0$ for $t \geq 0, t_{0} \geq 0$.

Let $n=m+p$, where $m, p$ are non-negative integers. Denote $x=(y, z)$, where $y=$ $\left(y_{1}, y_{2}, \ldots, y_{m}\right) \in \mathbb{R}^{m}, z=\left(z_{1}, z_{2}, \ldots, z_{p}\right) \in \mathbb{R}^{p}\|$.$\| any norm in \mathbb{R}^{n}$ and $\|.\|_{m}$ any norm in $\mathbb{R}^{m}$. Then, the system (2) could be written in the form

$$
\begin{aligned}
& { }_{t_{0}}^{c} D_{t}^{q} y_{i}(t)=f_{i}(t, y(t), z(t)), i=1,2, \ldots, m, \\
& { }_{t_{0}}^{c} D_{t}^{q} z_{j}(t)=f_{m+j}(t, y(t), z(t)), j=1,2, \ldots, p, \\
& y_{i}\left(t_{0}\right)=x_{0, i}, i=1,2, \ldots, m, \text { and } z_{j}\left(t_{0}\right)=x_{0, m+j}, j=1,2, \ldots, p
\end{aligned}
$$


Let the function $f \in C\left[\mathbb{R}_{+} \times \mathbb{R}^{n}, \mathbb{R}^{n}\right]$ be such that for any initial data $\left(t_{0}, x_{0}\right) \in \mathbb{R}_{+} \times \mathbb{R}^{n}$ the IVP for FrDE (2) has a solution $x\left(t ; t_{0}, x_{0}\right) \in C^{q}\left(\left[t_{0}, \infty\right), \mathbb{R}^{n}\right)$ (see, for example, $[24,27,31]$ for existence results of solutions of IVP for FrDE (2)).

The goal of the paper is to study the stability of the system FrDE (2) or its equivalent (3). Note the change of the initial time in the fractional differential equations reflects also on the fractional derivative and the type of equation (which is different than ordinary differential equations). In connection with this, we introduce the following definition:

Definition 2. The FrDE (3) is said to be

- $\quad$ stable if, for every $\epsilon>0$, there exist $\delta=\delta(\epsilon)>0$ such that, for any $x_{0} \in \mathbb{R}^{n}$, the inequality $\left\|x_{0}\right\|<\delta$ implies $\left\|x\left(t ; t_{0}, x_{0}\right)\right\|<\epsilon$ for $t \geq t_{0}$;

- $\quad$ stable w.r.t. $y$ if, for every $\epsilon>0$, there exists $\delta=\delta(\epsilon)>0$ such that for $x_{0} \in \mathbb{R}^{n}:\left\|x_{0}\right\|<\delta$ the inequality $\left\|y\left(t ; t_{0}, x_{0}\right)\right\|_{m}<\epsilon$ holds for $t \geq t_{0}$;

- $\quad$ attractive if there exists $\beta>0$ such that for every $\epsilon>0$ there exists $T=T(\epsilon)>0$ such that, for any $x_{0} \in \mathbb{R}^{n}$ with $\left\|x_{0}\right\|<\beta$, the inequality $\left\|x\left(t ; t_{0}, x_{0}\right)\right\|<\epsilon$ holds for $t \geq t_{0}+T$;

- $\quad$ attractive w.r.t. $y$ if there exists $\beta>0$ such that for every $\epsilon>0$ there exists $T=T(\epsilon)>0$ such that, for any $x_{0} \in \mathbb{R}^{n}$ with $\left\|x_{0}\right\|<\beta$, the inequality $\left\|y\left(t ; t_{0}, x_{0}\right)\right\|_{m}<\epsilon$ holds for $t \geq t_{0}+T$;

- asymptotically stable if the FrDE (2) is stable and attractive.

- $\quad$ asymptotically stable w.r.t. $y$ if the FrDE (2) is stable w.r.t. $y$ and attractive w.r.t. $y$.

Consider the following sets:

$$
\begin{aligned}
\mathcal{K} & =\left\{a \in C\left[\mathbb{R}_{+}, \mathbb{R}_{+}\right]: a \text { is strictly increasing and } a(0)=0\right\}, \\
B(\lambda) & =\left\{y \in \mathbb{R}^{m}:\|y\|_{m} \leq \lambda\right\}, \quad \lambda=\text { const }>0 .
\end{aligned}
$$

We will use the IVP for scalar Caputo FrDE of the form

$$
{ }_{t_{0}}^{c} D_{t}^{q} u(t)=g(t, u(t)), \quad t \geq t_{0}, \quad u\left(t_{0}\right)=u_{0},
$$

where $u, u_{0} \in \mathbb{R}, g: \mathbb{R}_{+} \times \mathbb{R} \rightarrow \mathbb{R}, g(t, 0) \equiv 0$ for $t \geq 0, t_{0} \geq 0$. We will assume that, for any initial data $\left(t_{0}, u_{0}\right) \in \mathbb{R}_{+} \times \mathbb{R}$, the IVP for the scalar FrDE (4) with $u\left(t_{0}\right)=u_{0}$ has a solution $u\left(t ; t_{0}, u_{0}\right) \in$ $C^{q}\left(\left[t_{0}, \infty\right), \mathbb{R}\right)$ (we will assume the existence of a maximal solution in Section 5 ). For some existence results, we refer $[24,27,31]$.

\section{Lyapunov Functions}

Definition 3. Let $t_{0}, T \in \mathbb{R}_{+}: t_{0}<T \leq \infty$, and $\Delta \subset \mathbb{R}^{n}, 0 \in \Delta$. We will say that the function $V(t, x):\left[t_{0}, T\right) \times \Delta \rightarrow \mathbb{R}_{+}$belongs to the class $\Lambda\left(\left[t_{0}, T\right), \Delta\right)$ if $V(t, x) \in C\left(\left[t_{0}, T\right) \times \Delta, \mathbb{R}_{+}\right)$is locally Lipschitzian with respect to its second argument and $V(t, 0) \equiv 0$.

In the literature, there are several types of derivatives of Lyapunov functions among solutions of fractional differential equations. We will present some of them:

I type: Let $x(t)$ be a solution of IVP for FrDE (2). Then, the Caputo fractional derivative of the Lyapunov function $V(t, x(t))$ is defined by

$$
{ }_{t_{0}}^{C} D_{t}^{q} V(t, x(t))=\frac{1}{\Gamma(1-q)} \int_{t_{0}}^{t}(t-s)^{-q} \frac{d}{d s}(V(s, x(s))) d s, \quad t \in\left(t_{0}, t_{0}+T\right) .
$$

It is used mainly for quadratic Lyapunov functions to study several stability properties of fractional differential equations (see, for example, [13]). This type of derivative is applicable for Lyapunov functions such that ${ }_{t_{0}}^{C} D_{t}^{q} V(t, x(t))$ exists (for example, continuously differentiable Lyapunov functions). 
II type: This type of derivative of $V(t, x)$ among FrDE (2) was introduced in [25]:

$$
D_{(2)}^{+} V(t, x)=\limsup _{h \rightarrow 0} \frac{1}{h^{q}}\left[V(t, x)-V\left(t-h, x-h^{q} f(t, x)\right], \quad t \in\left(t_{0}, t_{0}+T\right) .\right.
$$

The operator defined by (6) does not depend on the fractional order $q$ and it has no memory as is typical for fractional derivatives.

Remark 3. Let $x(t)$ be a solution of FrDE (2) for $t \geq t_{0}$. Then, $D_{(2)}^{+} V(t, x(t)) \neq{ }_{t_{0}}^{C} D_{t}^{q} V(t, x(t))$, $t \geq t_{0}$.

Now, let us recall the remark in [30] concerning definition (4) where $V\left(t-h, x-h^{q} f(t, x)\right)$ is defined by

$$
V\left(t-h, x-h^{q} f(t, x)\right)=\sum_{r=1}^{\left[\frac{t-t_{0}}{h}\right]}(-1)^{r+1}{ }_{q} C_{r} V\left(t-r h, x-h^{q} f(t, x)\right),
$$

where ${ }_{q} C_{r}=\frac{q(q-1) \ldots(q-r+1)}{r !}$ and $r$ is a natural number.

Applying this notation, we define the fractional derivative of the Lyapunov function by

$$
\begin{aligned}
& \mathcal{D}_{(2)}^{+} V\left(t, x ; t_{0}\right) \\
& =\limsup _{h \rightarrow 0} \frac{1}{h^{q}}\left[V(t, x)-\sum_{r=1}^{\left[\frac{t-t_{0}}{h}\right]}(-1)^{r+1}{ }_{q} C_{r} V\left(t-r h, x-h^{q} f(t, x)\right)\right] .
\end{aligned}
$$

The derivative (7) depends on the initial time $t_{0}$. The derivative (7) is called the Dini fractional derivative of the Lyapunov function. It is applicable for continuous Lyapunov functions.

Remark 4. In the general case, $\mathcal{D}_{(2)}^{+} V(t, x) \neq D_{(2)}^{+} V(t, x)$ (see Example 1, Case 2.1 and Case 2.2).

III type: for any $x \in \mathbb{R}^{n}$ the Caputo fractional Dini derivative of the Lyapunov function $V(t, x)$ among IVP for $\operatorname{FrDE}(2)$ with initial point $t_{0}$ and initial value $x_{0} \in \mathbb{R}^{n}$ is defined by:

$$
\begin{aligned}
& { }_{(2)}^{c} D_{+}^{q} V\left(t, x ; t_{0}, x_{0}\right)=\limsup _{h \rightarrow 0^{+}} \frac{1}{h^{q}}\left\{V(t, x)-V\left(t_{0}, x_{0}\right)\right. \\
& \left.-\sum_{r=1}^{\left[\frac{t-t_{0}}{h}\right]}(-1)^{r+1}{ }_{q} C_{r}\left(V\left(t-r h, x-h^{q} f(t, x)\right)-V\left(t_{0}, x_{0}\right)\right)\right\} \\
& \text { for } t \in\left(t_{0}, t_{0}+T\right) \text {, }
\end{aligned}
$$

or its equivalent

$$
\begin{aligned}
& \stackrel{c}{(2)} D_{+}^{q} V\left(t, x ; t_{0}, x_{0}\right) \\
& =\underset{h \rightarrow 0^{+}}{\limsup } \frac{1}{h^{q}}\left\{V(t, x)+\sum_{r=1}^{\left[\frac{t-t_{0}}{h}\right]}(-1)^{r}{ }_{q} C_{r} V\left(t-r h, x-h^{q} f(t, x)\right\}\right. \\
& \quad-\frac{V\left(t_{0}, x_{0}\right)}{\left(t-t_{0}\right)^{q} \Gamma(1-q)}, \quad \text { for } t \in\left(t_{0}, t_{0}+T\right) .
\end{aligned}
$$

The Caputo fractional Dini derivative (9) depends on both the fractional order $q$ and the initial data $\left(t_{0}, \psi\right)$ of IVP for FrDE (2). This type of derivative is close to the Caputo 
fractional derivative of a function. It is applicable for continuous Lyapunov functions (see, for example, [16,32]).

Remark 5. The equality ${ }_{(2)}^{c} D_{+}^{q} V\left(t, x ; t_{0}, x_{0}\right)=\mathcal{D}_{(2)}^{+} V\left(t, x ; t_{0}\right)-\frac{V\left(t_{0}, x_{0}\right)}{\left(t-t_{0}\right)^{q} \Gamma(1-q)}$ holds for any $t \in\left(t_{0}, t_{0}+T\right)$ and for any initial data $\left(t_{0}, x_{0}\right) \in \mathbb{R}_{+} \times \mathbb{R}^{n}$ :

$$
\begin{aligned}
& { }_{(2)}^{c} D_{+}^{q} V\left(t, x ; t_{0}, x_{0}\right)=\mathcal{D}_{(2)}^{+} V\left(t, x ; t_{0}\right), \quad \text { if } V\left(t_{0}, x_{0}\right)=0, \\
& { }_{(2)}^{c} D_{+}^{q} V\left(t, x ; t_{0}, x_{0}\right)<\mathcal{D}_{(2)}^{+} V\left(t, x ; t_{0}\right), \text { if } V\left(t_{0}, x_{0}\right)>0 .
\end{aligned}
$$

We will provide an example to illustrate the application of the above defined derivatives.

Example 1. Consider the scalar case $n=1$ and the Lyapunov function $V(t, x)=g(t) x^{2}$ where $g \in C^{1}\left(\left[t_{0}, \infty\right), \mathbb{R}_{+}\right)$.

Case 1. Let $x(t)$ be a solution of the IVP for FrDE (2). Then, according to Equation (5), the Caputo fractional derivative of the Lyapunov function is

$$
{ }_{t_{0}}^{C} D_{t}^{q} V(t, x(t))=\frac{2}{\Gamma(1-q)} \int_{t_{0}}^{t}(t-s)^{-q} \frac{d}{d s}\left(g(s) x^{2}(s)\right) d s .
$$

In the general case, the above integral is difficult to solve and also obtaining upper bounds might not be possible.

In the special case $g(t) \equiv 1$, i.e., we consider the quadratic Lyapunov function, we could apply Lemma 1 and obtain

$$
{ }_{t_{0}}^{C} D_{t}^{q} V(t, x(t)) \leq 2 x(t){ }_{t_{0}}^{C} D_{t}^{q} x(t)=2 x(t) f(t, x(t)) .
$$

Case 2. Second type of derivative. Let $x, x_{0} \in \mathbb{R}$ and $t>t_{0}$.

Case 2.1: From formula (6) we obtain

$$
\begin{aligned}
& D_{(2)}^{+} V(t, x)=\limsup _{h \rightarrow 0} \frac{1}{h^{q}}\left[g(t) x^{2}-g(t-h)\left(x-h^{q} f(t, x)\right)^{2}\right] \\
& =x^{2} \limsup _{h \rightarrow 0} \frac{g(t)-g(t-h)}{h} h^{1-q} \\
& +2 x f(t, x) \limsup _{h \rightarrow 0} g(t-h)-(f(t, x))^{2} \limsup _{h \rightarrow 0} h^{q} g(t-h) \\
& =2 x g(t) f(t, x) .
\end{aligned}
$$

The derivative $D_{(2)}^{+} V(t, x)$ does not depend on both the order $q$ of the fractional derivative and initial time $t_{0}$.

Case 2.2: Dini fractional derivative. Apply formula (7) and obtain

$$
\begin{aligned}
& \mathcal{D}_{(2)}^{+} V\left(t, x ; t_{0}\right)=x^{2} \limsup _{h \rightarrow 0} \frac{1}{h^{q}} \sum_{r=0}^{\left[\frac{t-t_{0}}{h}\right]}(-1)^{r}{ }_{q} C_{r} g(t-r h)+2 x g(t) f(t, x) \\
& -2 x f(t, x) \limsup _{h \rightarrow 0} \sum_{r=0}^{\left[\frac{t-t_{0}}{h}\right]}(-1)^{r}{ }_{q} C_{r} g(t-r h) \\
& -(f(t, x))^{2} \limsup _{h \rightarrow 0} h^{q} \sum_{r=1}^{\left[\frac{t-t_{0}}{h}\right]}(-1)^{r}{ }_{q} C_{r} g(t-r h) \\
& =x^{2}{ }_{t_{0}}^{R L} D_{t}^{q} g(t)+2 x g(t) f(t, x) .
\end{aligned}
$$


In the case when the Lyapunov function is a fractional derivative of the Lyapunov function, which is a quadratic function, we get

$$
\mathcal{D}_{(2)}^{+} V\left(t, x ; t_{0}\right)=2 x f(t, x)+\frac{x^{2}}{\left(t-t_{0}\right)^{q} \Gamma(1-q)} .
$$

The Dini fractional derivative depends on both the fractional order $q$ and initial time. Similar to fractional derivatives, it has a memory.

Case 2.3: Caputo fractional Dini derivative. According to Remark 5 and Case 2.2, we get

$$
{ }_{(2)}^{c} D_{+}^{q} V\left(t, x ; t_{0}, x_{0}\right)=x^{2}{ }_{t_{0}}^{R L} D_{t}^{q} g(t)+2 g(t) x f(t, x)-\frac{g\left(t_{0}\right) x_{0}^{2}}{\left(t-t_{0}\right)^{q} \Gamma(1-q)} .
$$

In the special case of the quadratic Lyapunov function, i.e., $g(t) \equiv 1$, we obtain

$$
{ }_{(2)}^{c} D_{+}^{q} V\left(t, x ; t_{0}, x_{0}\right)=2 x f(t, x)+\frac{x^{2}-x_{0}^{2}}{\left(t-t_{0}\right)^{q} \Gamma(1-q)} .
$$

The Caputo fractional Dini derivative depends on both the fractional order $q$ and initial data, which is typical for the Caputo fractional operator.

Now let us consider the case $q=1$, i.e., the case of ordinary derivatives. The well known Dini derivative of the Lyapunov function $V(t, x)=g(t) x^{2}$ is $V_{+}^{\prime}(t, x)=2 g(t) x f(t, x)+x^{2}(g(t))^{\prime}$. In the special case of a quadratic Lyapunov function, i.e., $g(t) \equiv 1$, all derivatives $D_{(2)}^{+} V(t, x)$, $\mathcal{D}_{(2)}^{+} V\left(t, x ; t_{0}\right)$ and ${ }_{(2)}^{c} D_{+}^{q} V\left(t, x ; t_{0}, x_{0}\right)$ are equal to $2 x f(t, x)$ and coincide with the Dini derivative $V_{+}^{\prime}(t, x)$. Therefore, for quadratic Lyapunov functions, it is natural to consider the above given derivatives as extensions of the ordinary case $q=1$. In the general case, $g(t) \not \equiv 1$ only the Dini fractional derivative $\mathcal{D}_{(2)}^{+} V\left(t, x ; t_{0}\right)$ and the Caputo fractional Dini derivative satisfies $\mathcal{D}_{(2)}^{+} V\left(t, x ; t_{0}\right)=$ ${ }_{(2)}^{c} D_{+}^{q} V\left(t, x ; t_{0}, x_{0}\right)=2 g(t) x f(t, x)+x^{2}{ }_{t_{0}}^{R L} D_{t}^{q} g(t)$. Therefore, in the general case when the Lyapunov functions depend implicitly on the time variable, it seems both Dini fractional derivative and Caputo fractional Dini derivative are extensions of the ordinary case $q=1$ where the ordinary derivative of $g(t)$ is replaced by the R-L fractional derivative.

From the literature, we note that one of the sufficient conditions for stability is connected with the sign of the derivative of the Lyapunov function of the equation.

Example 2. Consider the IVP for the scalar linear FrDE

$$
\begin{aligned}
& { }_{0}^{C} D_{t}^{0.9} x(t)=-\left(0.5 \frac{0^{R L} D^{0.9}\left(\sin ^{2}(t)+0.1\right)}{\sin ^{2}(t)+0.1}\right) x(t) \text { for } t>0, \\
& x(0)=1 .
\end{aligned}
$$

Denote $G(t)=-0.5 \frac{{ }_{0}^{R L} D^{0.9}\left(\sin ^{2}(t)+0.1\right)}{\sin ^{2}(t)+0.1}$

Using $\sin ^{2}(t)+0.1=0.6-0.5 \cos (2 t)$, we get ${ }_{0}^{R L} D^{0.9}\left(\sin ^{2}(t)+0.1\right)=\frac{0.6}{t^{0.9} \Gamma(0.1)}-$ $\frac{P F Q\left[\{1\},\{0.55,1.05\},-t^{2}\right]}{2 t^{0.9} \Gamma(0.1)}+4 \frac{t^{1.1} P F Q\left[\{2\},\{1.55,2.05\},-t^{2}\right]}{\Gamma(3.1)}$, where PFQ is the generalized hypergeometric functions.

Consider the Lyapunov function $V(t, x)=\left(\sin ^{2}(t)+0.1\right) x^{2}$.

Case 1: Caputo fractional derivative. According to Example 1, Case 1, the fractional derivative of this function $V$ is difficult to obtain so it is difficult to discuss its sign.

Case 2: According to Example 1, Case 2.1, for any $x \in \mathbb{R}$, we obtain

$$
D_{(14)}^{+} V(t, x)=2 x^{2}\left(\sin ^{2}(t)+0.1\right) G(t) .
$$


The sign of the function $G(t)$ is changeable (see Figure 1) and, therefore, the sign of the derivative $D_{(14)}^{+} V(t, x)$ is changeable.

Case 3: Dini fractional derivative. According to Example 1, Case 2.2 for any $x \in \mathbb{R}$, we apply formula (7) and obtain

$$
\mathcal{D}_{(14)}^{+} V(t, x ; 0)=2 x^{2} G(t)\left(\sin ^{2}(t)+0.1\right)+x_{0}^{2}{ }_{0}^{R L} D^{0.9}\left(\sin ^{2}(t)+0.1\right)=0 .
$$

Case 4: Caputo fractional Dini derivative. According to Remark 5 and Case 2.3 of Example 1, the inequality

$$
{ }_{(14)}^{c} D_{+}^{0.9} V(t, x ; 0,1)=\mathcal{D}_{(14)}^{+} V(t, x ; 0)-\frac{0.1}{t^{0.9} \Gamma(0.1)}=-\frac{0.1}{t^{0.9} \Gamma(0.1)}<0
$$

holds.

Therefore, for (14), both the Dini fractional derivative and the Caputo fractional Dini derivative seem to be more applicable than the Caputo fractional derivative of the Lyapunov function.

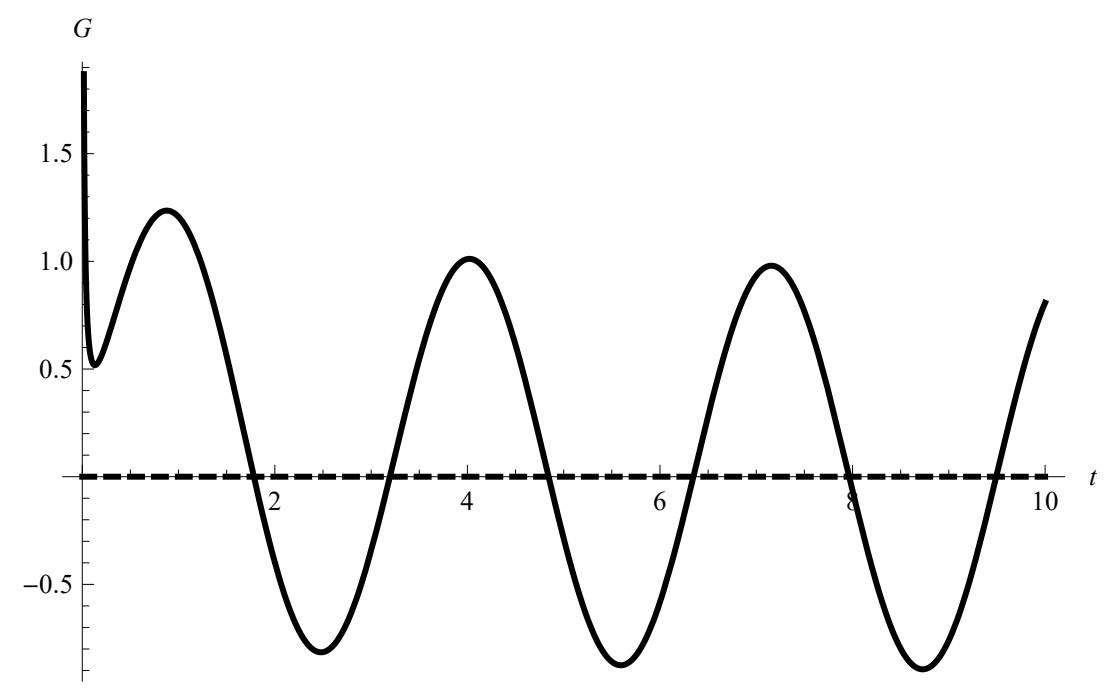

Figure 1. Example 2: Graph of the function $G(t)$.

Remark 6. The above example notes that the quadratic function for studying stability properties of fractional differential equations might not be successful (especially when the right-hand side depends directly on the time variable). Formula (4) is not appropriate for applications to fractional equations. The most general derivatives for non-homogenous fractional differential equations are Dini fractional derivatives and Caputo fractional Dini derivatives.

In some papers (see, for example, [33,34] (Theorem 1)), the authors use the equality ${ }_{t_{0}}^{C} D_{t}^{q} V(t, x(t))=D_{(2)}^{+} V(t, x(t))$, where $x(t)$ is a solution of (2). This equality is not true and we will demonstrate this on a particular Lyapunov function and Caputo fractional deferential equation.

Example 3. Consider the IVP for the scalar linear FrDE

$$
{ }_{0}^{C} D_{t}^{0.9} x(t)=G(t) x(t) \text { for } t>0, \quad x(0)=1,
$$

where $G(t)=\frac{10^{0} \cdot 1}{(t+1) \Gamma(0.9)}$. The function $x(t)=t+1$ is a solution of the FrDE (15).

Consider the function $V(t, x)=\frac{t}{t+1} x^{2}$. 
Case 1: Caputo fractional derivative. According to (5) and using $V(t, x(t))=t(t+1)$, we get

$$
{ }_{0}^{C} D_{t}^{0.9} V(t, x(t))=\frac{1}{\Gamma(0.1)} \int_{0}^{t}(t-s)^{-0.9} \frac{d}{d s}(s(s+1)) d s=\frac{1}{\Gamma(0.1)}\left(10 t^{0.1}+\frac{2}{0.11} t^{1.1}\right) .
$$

Case 2. According to Example 1, Case 2.1 and Equation (11), we obtain

$$
D_{(15)}^{+} V(t, x(t))=2(t+1) \frac{t}{t+1} \frac{10 t^{0.1}}{(t+1) \Gamma(0.9)}(t+1)=\frac{20 t^{1.1}}{\Gamma(0.9)}
$$

From Equations (16) and (17), it is clear that equality ${ }_{t_{0}}^{C} D_{t}^{q} V(t, x(t))=D_{(15)}^{+} V(t, x(t))$ is not true (see Figure 2) and the replacement of the Caputo fractional derivative ${ }_{t_{0}}^{C} D_{t}^{q} V(t, x(t))$ in any proof by $D_{(2)}^{+} V(t, x(t))$ is not correct.

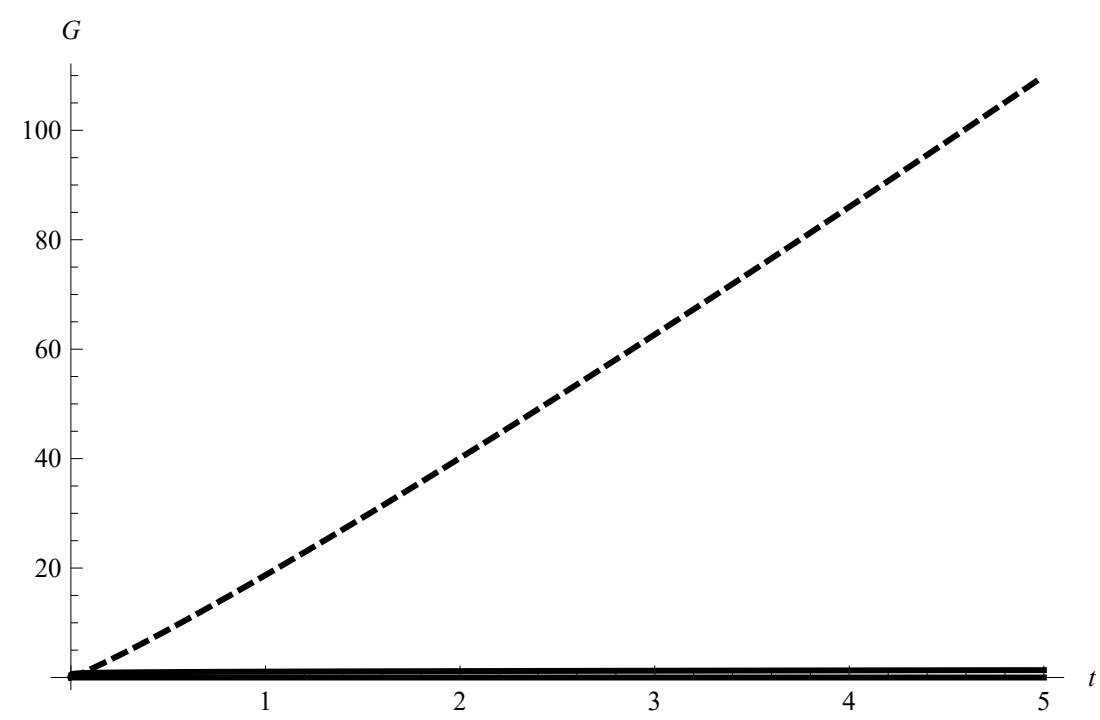

Figure 2. Example 3. Graph of the both derivatives of $V=\frac{t}{t+1} x^{2}$.

\section{Comparison Results for Scalar FrDE}

Many results about stability by the second Lyapunov method and fractional derivatives of Lyapunov functions are known in the literature. For example, in [13], the Mittag-Leffler stability as well as asymptotic stability is studied and several sufficient conditions using Caputo fractional derivatives of Lyapunov functions are obtained. In [35], the Riemann-Liouville fractional derivative of Lyapunov functions is applied which allow less restrictions for the Lyapunov functions from $C_{1-p}((a, b], \mathbb{R})=\left\{u \in C((a, b], \mathbb{R}): u(t)(t-a)^{1-p} \in C((a, b], \mathbb{R})\right\}$.

In connection with Example 1, Case 2.1 and Example 3, we will not use the derivative of Lyapunov functions given by (6).

We will provide and use some comparison results for Caputo fractional Dini derivatives.

Lemma 2 (Ref. [16]). (Comparison result). Assume the following conditions are satisfied:

1. The function $x^{*}(t)=x\left(t ; t_{0}, x_{0}\right), x^{*} \in C^{q}\left(\left[t_{0}, T\right], \Delta\right)$ is a solution of the FrDE (2) where $\Delta \subset \mathbb{R}^{n}, 0 \in \Delta$, $t_{0}, T \in \mathbb{R}_{+}: t_{0}<T$ are given constants, $x_{0} \in \Delta$.

2. The function $g \in C\left(\left[t_{0}, T\right] \times \mathbb{R}, \mathbb{R}\right)$.

3. The function $V \in \Lambda\left(\left[t_{0}, T\right], \Delta\right)$ and for any $t \in\left[t_{0}, T\right]$ the inequality

$$
{ }_{(2)}^{c} D_{+}^{q} V\left(t, x^{*}(t) ; t_{0}, x_{0}\right) \leq g\left(t, V\left(t, x^{*}(t)\right)\right)
$$


holds.

4. The function $u^{*}(t)=u\left(t ; t_{0}, u_{0}\right), u^{*} \in C^{q}\left(\left[t_{0}, T\right], \mathbb{R}\right)$, is the maximal solution of the initial value problem (4).

Then, the inequality $V\left(t_{0}, x_{0}\right) \leq u_{0}$ implies $V\left(t, x^{*}(t)\right) \leq u^{*}(t)$ for $t \in\left[t_{0}, T\right]$.

If $g(t, x) \equiv 0$ in Lemma 2, we obtain the following result:

Corollary 1 (Ref .[16]). Assume:

1. The condition 1 of Lemma 2 is satisfied.

2. The function $V \in \Lambda\left(\left[t_{0}, T\right], \Delta\right)$ and for any $t \in\left[t_{0}, T\right]$ the inequality

$$
{ }_{(2)}^{c} D_{+}^{q} V\left(t, x^{*}(t) ; t_{0}, x_{0}\right) \leq 0
$$

holds.

Then, for $t \in\left[t_{0}, T\right]$, the inequality $V\left(t, x^{*}(t)\right) \leq V\left(t_{0}, x_{0}\right)$ holds.

Corollary 2 (Ref. [16]). Assume:

1. The condition 1 of Lemma 2 is satisfied.

2. The function $V \in \Lambda\left(\left[t_{0}, T\right], \Delta\right)$ and for any $t \in\left[t_{0}, T\right]$ the inequality

$$
{ }_{(2)}^{c} D_{+}^{q} V\left(t, x^{*}(t) ; t_{0}, x_{0}\right) \leq-c V\left(t, x^{*}(t)\right)
$$

holds.

Then, for $t \in\left[t_{0}, T\right]$, the inequality $V\left(t, x^{*}(t)\right) \leq V\left(t_{0}, x_{0}\right) E_{q}\left(-c\left(t-t_{0}\right)^{q}\right)$ holds.

Remark 7. The result of Lemma 2 is also true on the half line.

Lemma 3 (Ref. [16]). Assume:

1. The condition 1 of Theorem 1 is satisfied.

2. The function $V \in \Lambda\left(\left[t_{0}, T\right], \Delta\right)$ and for any $t \in\left[t_{0}, T\right]$ the inequality

$$
{ }_{(2)}^{c} D_{+}^{q} V\left(t, x^{*}(t) ; t_{0}, x_{0}\right) \leq-c\left(\left\|x^{*}(t)\right\|\right)
$$

holds where $c \in \mathcal{K}$.

Then for $t \in\left[t_{0}, T\right]$ the inequality

$$
V\left(t, x^{*}(t)\right) \leq V\left(t_{0}, x_{0}\right)-\frac{1}{\Gamma(q)} \int_{t_{0}}^{t}(t-s)^{q-1} c\left(\left\|x^{*}(s)\right\|\right) d s
$$

holds.

\section{Stability Results in the Part of Variables for Fractional Differential Equations}

We are now in a position to study stability properties w.r.t. part of the variables of the nonlinear Caputo fractional differential equations of type (2). We will apply the Caputo fractional Dini derivative of Lyapunov functions. We do not use solutions to the system (2) in the sufficient conditions.

Theorem 1. Let the following conditions be satisfied:

1. The function $g \in C\left(\left[t_{0}, \infty\right) \times \mathbb{R}, \mathbb{R}\right)$. 
2. There exists a function $V \in \Lambda\left(\left[t_{0}, \infty\right), \Delta\right): \Delta=\left\{x=(y, z) \in \mathbb{R}^{n}: y \in B(\lambda)\right\}$ such that

(i) for any $x_{0} \in \mathbb{R}^{n}$ the inequality

$$
{ }_{(3)}^{c} D_{+}^{q} V\left(t, x ; t_{0}, x_{0}\right) \leq g(t, V(t, x)), \quad t \geq t_{0}, x \in \mathbb{R}^{n}: y \in B(\lambda)
$$

(ii) $\quad b\left(\|y\|_{m}\right) \leq V(t, x) \leq a(\|x\|)$ for $t \geq t_{0}, x \in \mathbb{R}^{n}: y \in B(\lambda)$ where $a, b \in \mathcal{K}$.

3. The scalar FrDE (4) is stable.

Then, the FrDE (3) is stable w.r.t. y.

Proof. Let $\epsilon>0$ be an arbitrary number. According to Condition 3, there exists $\delta=\delta(\epsilon)>0$ such that for $u_{0} \in \mathbb{R}:\left|u_{0}\right|<\delta$ the inequality

$$
\left|u\left(t ; t_{0}, u_{0}\right)\right|<b(\epsilon)
$$

holds for $t \geq t_{0}$ where $u\left(t ; t_{0}, u_{0}\right)$ is a solution of scalar FrDE (4) with initial data $\left(t_{0}, u_{0}\right)$.

Let $\delta_{1}<\min \left\{\varepsilon, b(\varepsilon\}\right.$. There exists $\delta_{2}=\delta_{2}(\varepsilon)>0$ such that the inequality $s<\delta_{2}$ implies $a(s)<\delta_{1}$. Let $\delta<\min \left\{\varepsilon, \delta_{2}\right\}$. Choose $x_{0}:\left\|x_{0}\right\|<\delta$ and consider $x^{*}(t)=x\left(t ; t_{0}, x_{0}\right), t \geq t_{0}$. We will prove

$$
\left\|y\left(t ; t_{0}, x_{0}\right)\right\|_{m}<\varepsilon, \quad t \geq t_{0} .
$$

Note the inequalities $\left\|y_{0}\right\|_{m} \leq\left\|x_{0}\right\|<\delta \leq \varepsilon$ hold. Assume inequality (21) is not true. Therefore, there exists $t^{*}>t_{0}$ such that

$$
\left\|y\left(t^{*} ; t_{0}, x_{0}\right)\right\|_{m}=\varepsilon \text { and }\left\|y\left(t ; t_{0}, x_{0}\right)\right\|_{m}<\varepsilon, \quad t \geq\left[t_{0}, t^{*}\right) .
$$

Therefore, for the solution $x\left(t ; t_{0}, x_{0}\right)$, the component $y\left(t ; t_{0}, x_{0}\right) \in B(\lambda)$ on $\left[t_{0}, t^{*}\right]$.

Consider the solution $u\left(t ; t_{0}, u_{0}^{*}\right)$ of the scalar FrDE (4) with initial data $\left(t_{0}, u_{0}^{*}\right): u_{0}^{*}=$ $V\left(t_{0}, x_{0}\right) \leq a\left(\left\|x_{0}\right\|\right)<a(\delta) \leq a\left(\delta_{1}\right)$. Therefore, the inequality (20) holds. According to Lemma 2 with $T=t^{*}, \Delta=\left\{x=(y, z) \in \mathbb{R}^{n}: y \in B(\lambda)\right\}$, condition 2(ii) and inequalities (22), we get $b(\varepsilon)=b\left(\left\|y\left(t^{*} ; t_{0}, x_{0}\right)\right\|_{m}\right) \leq V\left(t^{*}, x\left(t ; t_{0}, x_{0}\right)\right) \leq u\left(t^{*} ; t_{0}, u_{0}^{*}\right)<b(\varepsilon)$.

The contradiction proves inequality (21).

Corollary 3. Let condition 2 of Theorem 1 be satisfied where the inequality (19) is replaced by ${ }_{\text {(3) }}^{c} D_{+}^{q} V\left(t, x ; t_{0}, x_{0}\right) \leq 0, \quad t \in \mathbb{R}_{+}, x=(y, z) \in \mathbb{R}^{n} y \in B(\lambda)$. Then, $\operatorname{FrDE}$ (3) is stable w.r.t. $y$.

Now, we present some sufficient conditions for the uniform asymptotic stability w.r.t. part of the variables.

Theorem 2. Assume there exists a function $V \in \Lambda\left(\left[t_{0}, \infty\right), \Delta\right): \Delta=\left\{x=(y, z) \in \mathbb{R}^{n}: y \in B(\lambda)\right\}$ such that

(i) for any $x_{0} \in \mathbb{R}^{n}$ the inequality

$$
{ }_{(2)}^{c} D_{+}^{q} V\left(t, x ; t_{0}, x_{0}\right) \leq-c(\|x\|), \quad t \in \mathbb{R}_{+}, x \in \mathbb{R}^{n}: y \in B(\lambda) \subset \mathbb{R}^{m}
$$

holds where $c \in \mathcal{K}$;

(ii) $b\left(\|y\|_{m}\right) \leq V(t, x) \leq a(\|x\|)$ for $t \in \mathbb{R}_{+}, x=(x, y) \in \mathbb{R}^{n}: y \in B(\lambda) \subset \mathbb{R}^{m}$, where $a, b \in \mathcal{K}$.

Then, the FrDE (3) is asymptotically stable w.r.t. $y$.

Proof. According to Corollary 3, the FrDE (3) is stable w.r.t. $y$. Therefore, for the number $\lambda>0$, there exists $\alpha=\alpha(\lambda) \in(0, \lambda)$ such that for any $\tilde{x}_{0} \in \mathbb{R}^{n}$ the inequality $\left\|\tilde{x}_{0}\right\|<\alpha$ implies

$$
\left\|y\left(t ; t_{0}, \tilde{x}_{0}\right)\right\|_{m}<\lambda \text { for } t \geq \tilde{t}_{0},
$$


where $x\left(t ; t_{0}, \tilde{x}_{0}\right)$ is a solution of (2) with initial data $\left(t_{0}, \tilde{x}_{0}\right)$ and $x=(y, z), y\left(t ; t_{0}, \tilde{x}_{0}\right) \in \mathbb{R}^{m}$.

Now, we will prove the uniformly attractivity of the zero solution. Let $\varepsilon \in(0, \lambda]$ be an arbitrary number and choose $\gamma>0: a(\gamma)<b(\varepsilon)$. Choose $T=T(\varepsilon)>0$ such that $T>\sqrt[q]{q \Gamma(q) \frac{a(\alpha)}{c(\gamma)}}$.

Let $\left\|x_{0}\right\|<\alpha$. Then, the inequality (24) holds for the solution $x\left(t ; t_{0}, x_{0}\right)$ of (2) with initial data $\left(t_{0}, x_{0}\right)$ and $x=(y, z), y\left(t ; t_{0}, x_{0}\right) \in \mathbb{R}^{m}$, i.e., $y\left(t ; t_{0}, x_{0}\right) \in B(\lambda)$ for $t \geq t_{0}$. We will prove that

$$
\left\|x\left(t ; t_{0}, x_{0}\right)\right\|<\gamma \text { for } t \geq t_{0}+T .
$$

Assume that $\left\|x\left(t ; t_{0}, x_{0}\right)\right\| \geq \gamma$ on the entire interval $\left[t_{0}, t_{0}+T\right]$. Then, from Lemma 3 applied to the interval $\left[t_{0}, t_{0}+T\right]$, we obtain the inequality

$$
\begin{aligned}
V\left(t_{0}+T, x\left(t_{0}+T ; t_{0}, x_{0}\right)\right) & \leq V\left(t_{0}, x_{0}\right)-\frac{1}{\Gamma(q)} \int_{t_{0}}^{t_{0}+T}\left(t_{0}+T-s\right)^{q-1} c\left(\left\|x\left(s ; t_{0}, x_{0}\right)\right\|\right) d s \\
& \leq a\left(\left\|x_{0}\right\|\right)-\frac{c(\gamma)}{\Gamma(q)} \frac{T^{q}}{q}<0 .
\end{aligned}
$$

Therefore, there exists a point $t^{*} \in\left[t_{0}, t_{0}+T\right]$ such that $\left\|y\left(t^{*} ; t_{0}, x_{0}\right)\right\|_{m}<\gamma$. According to Lemma 3 for $t \geq t^{*}$, we have

$$
V\left(t, x\left(t ; t_{0}, x_{0}\right)\right) \leq V\left(t^{*}, x\left(t^{*} ; t_{0}, x_{0}\right)\right)-\frac{1}{\Gamma(q)} \int_{t^{*}}^{t}(t-s)^{q-1} c\left(\left\|x\left(s ; t_{0}, x_{0}\right)\right\|\right) d s \leq V\left(t^{*}, x\left(t^{*} ; t_{0}, x_{0}\right)\right) .
$$

Therefore, for $t \geq t^{*}$, the inequalities

$$
b\left(\left\|y\left(t ; t_{0}, x_{0}\right)\right\|_{m}\right) \leq V\left(t, x\left(t ; t_{0}, x_{0}\right)\right) \leq V\left(t^{*}, x\left(t^{*} ; t_{0}, x_{0}\right)\right) \leq a\left(\left\|x\left(t^{*} ; t_{0}, x_{0}\right)\right\|\right) \leq a(\gamma)<b(\varepsilon)
$$

hold.

Remark 8. Note that, in the case of continuously differentiable Lyapunov function, their Caputo fractional derivatives are applied to study stability w.r.t. part of the variables in [36].

Remark 9. Note the above results for stability w.r.t. part of variables are generalizations of the stability results with $n=m[16])$.

\section{Applications}

Now, we illustrate our theory on examples. We will use both Caputo fractional derivatives and Caputo fractional Dini derivatives of Lyapunov functions in particular FrDE.

Example 4. Let $n=m=1$ and consider the IVP for the scalar linear FrDE (14).

If we apply the quadratic Lyapunov function $V(t, x)=x^{2}$, then, according to Example 1, Case 1 ${ }_{0}^{C} D_{t}^{q} V(t, x(t)) \leq 2 x^{2}(t) G(t)$ and its sign is changeable (see Example 2 and Figure 1). Therefore, the sufficient conditions in [36] are not applicable. If we use non-quadratic Lyapunov function, then obtaining the Caputo fractional derivative is difficult and again the sufficient conditions using this type of derivatives are not useful.

Now, consider the function $V_{1}(t, x)=\left(\sin ^{2}(t)+0.1\right) x^{2}$ and its Caputo fractional Dini derivative. According to Example 2, Case 3, the inequality ${ }_{(14)}^{c} D_{+}^{0.9} V_{1}\left(t, x ; 0, x_{0}\right)=-\frac{0.1 x_{0}^{2}}{t^{0.9} \Gamma(0.1)}<0$ holds. According to Corollary 3 the FrDE (14) is stable.

This example shows that, in the case of nonlinear non autonomous fractional differential equations, non-quadratic Lyapunov functions with their Caputo fractional Dini derivatives may be useful. 
Example 5. Consider the following initial value problem for the system of fractional differential equations:

$$
\begin{array}{lrl}
{ }_{0}^{c} D^{0.5} x(t)=G(t) x(t)+F(t) x^{3}(t) \sin ^{2}(y(t)), & t>0, & x(0)=x_{0}, \\
{ }_{0}^{c} D^{0.5} y(t)=f(t, x(t), y(t)), \quad t>0, & y(0)=y_{0},
\end{array}
$$

where $x, y \in \mathbb{R}, G(t)=\frac{-0.125-\frac{1}{\sqrt{ } \Gamma \Gamma(0.5)}-2^{-1.5} \cos \left(2 t+\frac{\pi}{4}\right)}{1+\cos ^{2}(t)}, F(t)=\frac{0.25}{\sqrt{t} \Gamma(0.5)\left(1+\cos ^{2}(t)\right)}$.

Case 1. Consider the quadratic Lyapunov function $V(t, x, y)=x^{2}$ for $x \in B(1)=\{x \in \mathbb{R}:|x| \leq 1\}$. Case 1.1. The Caputo fractional derivative of $V$ satisfies

$$
{ }_{0}^{C} D_{t}^{0.5} V(t, x(t), y(t)) \leq 2 x^{2}(t) G(t)+2 x^{4}(t) F(t)\left(\sin ^{2}(y(t)) \leq 2 x^{2}(t)(G(t)+F(t)) .\right.
$$

Since the sign of the function $G(t)+F(t)$ is changeable (see Figure 3), the results obtained in [36] are not applicable.

Case 1.2. Dini fractional derivative. Similar to Example 1, Case 3, we obtain

$$
\begin{aligned}
\mathcal{D}_{(27)}^{+} V(t, x, y ; 0) & =2 x^{2} G(t)+2 x^{4}(t) F(t)\left(\sin ^{2}(y(t))+\left(x^{2}-\left(x_{0}\right)^{2}\right) \frac{t^{-0.5}}{\Gamma(0.5)}\right. \\
& \leq x^{2}\left(G(t)+F(t)+\frac{t^{-0.5}}{\Gamma(0.5)\left(1+\cos ^{2}(t)\right)}\right) \\
& =x^{2}\left(\frac{g(t)}{1+\cos ^{2}(t)}\right)
\end{aligned}
$$

where $g(t)=-0.125+0.25 \frac{1}{t^{0.5} \Gamma(0.5)}-2^{-1.5} \cos \left(2 t+\frac{\pi}{4}\right)$. Since the sign of the function $g(t)$ changes, so does the fractional derivative of $V$ (see Figure 4). Therefore, the quadratic function is not applicable to the fractional equation (27).

Case 2. Consider the Lyapunov function $V_{1}(t, x, y)=\left(1+\cos ^{2}(t)\right) x^{2}$.

Case 2.1. The Caputo derivative of the Lyapunov function $V_{1}(t, x(t), y(t))$ is difficult to obtain so we will not use it.

Case 2.2. Dini fractional derivative. According to Example 1, Case 3 and the equalities $\cos ^{2}(t)=$ $0.5+0.5 \cos (2 t)$ and ${ }_{0}^{c} D^{0.5}(\cos (2 t))=2^{0.5} \cos \left(2 t+\frac{\pi}{4}\right)$, we obtain

$$
\begin{aligned}
& \mathcal{D}_{(27)}^{+} V(t, x, y ; 0)=x^{2}\left(\frac{1.5}{t^{0.5} \Gamma(0.5)}+2^{-0.5} \cos \left(2 t+\frac{\pi}{4}\right)\right) \\
& \quad+2 x^{2}(G(t)+F(t))\left(1+\cos ^{2}(t)\right) \\
& =-0.25 x^{2} .
\end{aligned}
$$

According to Remark 5 and Theorem 1, the FrDE (27) is stable w.r.t. the variable $x$.

Note that the $\operatorname{FrDE}(27)$ might not be stable w.r.t. the other variable $y$ (if, for example, $f(t, x, y)=y$ ).

Fractional models describe an epidemic diseases that are more realistic because the fractional-order differential equation systems reproduce with more efficacy reality [37]. Usually, in epidemic models, all parameters such as the death removal rate, the infective class at a per capita constant rate, the number of contacts per infective, per day, which result in infection, are positive constants. Now, we will consider one SIR fractional model with time variable coefficients to illustrate our results. 


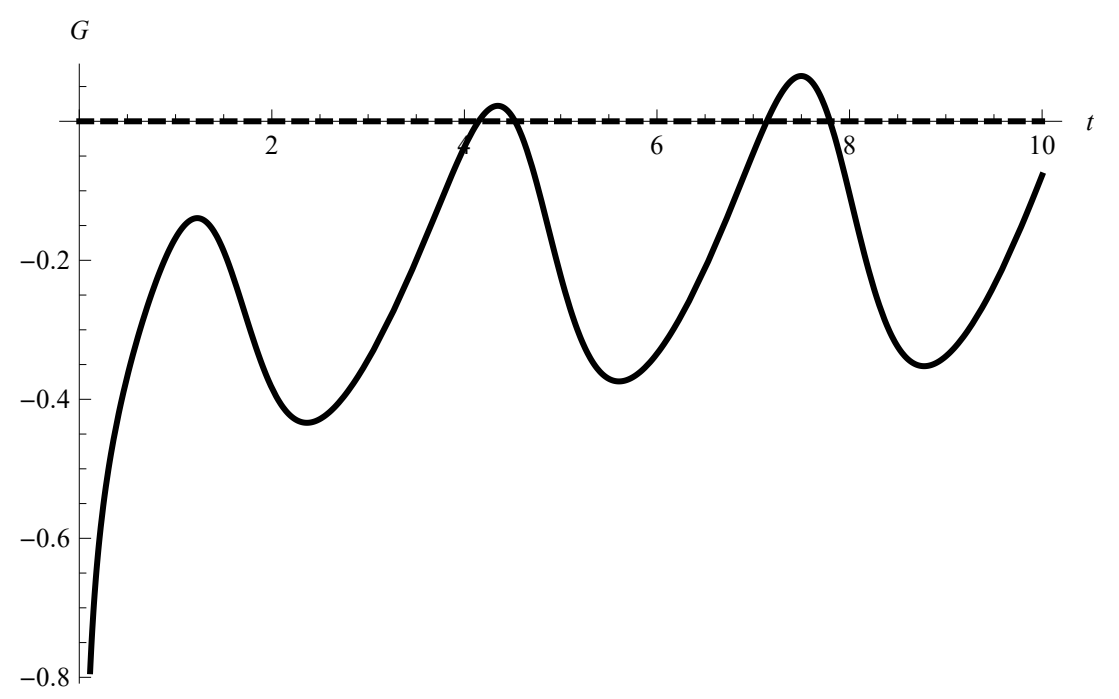

Figure 3. Graph of the function $\frac{-0.125-\frac{0.75}{\sqrt{t T}(0.5)}-2^{-1.5} \cos \left(2 t+\frac{\pi}{4}\right)}{1+\cos ^{2}(t)}$.

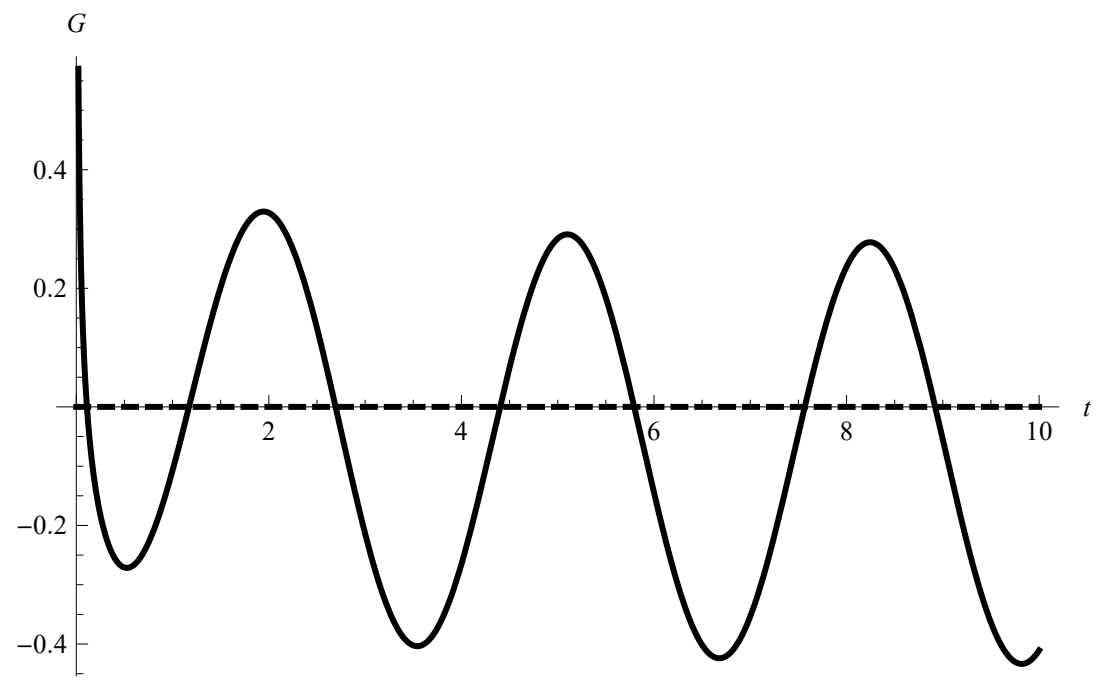

Figure 4. Example 5. Graph of the function $g(t)$.

Example 6. Consider the following SIR fractional model:

$$
\begin{aligned}
& { }_{0}^{c} D^{0.5} S(t)=\mu(t)-\lambda(t) S I-\mu(t) S, \quad t>0, \\
& { }_{0}^{c} D^{0.5} I(t)=\lambda(t) S I-(\mu(t)+\gamma(t)) I \quad t>0,
\end{aligned}
$$

where $\mu(t)=\frac{0.125+\frac{1}{\sqrt{t} \Gamma(0.5)}}{1+\cos ^{2}(t)}$ is the death removal rate, $\gamma(t)=\frac{2^{-1.5} \cos \left(2 t+\frac{\pi}{4}\right)}{1+\cos ^{2}(t)}$ is the infective class at a per capita rate, and $\lambda(t)=\frac{0.25}{\sqrt{t} \Gamma(0.5)\left(1+\cos ^{2}(t)\right)}>0$ is the number of contacts per infective at time $t$ which result in infection.

The point $(1,0)$ is an equilibrium point of $(29)$.

We will apply some Lyapunov functions to study stability properties. 
Case 1. Consider the quadratic Lyapunov function $V(t, S, I)=I^{2}$. Then, using Example 1, Case 1, inequalities (10) and $|S| \leq 1$ we obtain for the Caputo fractional derivative of $V$ :

$$
{ }_{0}^{C} D_{t}^{0.5} V(t, S(t), I(t)) \leq 2 I(t)(\lambda(t) S(t) I(t)-(\mu(t)(t)+\gamma(t)) I(t)) \leq 2(-\mu(t)-\gamma(t)+\lambda(t)) I^{2}(t) .
$$

Since the sign of the function $-\mu(t)-\gamma(t)+\lambda(t)=\frac{-0.125-\frac{1}{\sqrt{t \Gamma}(0.5)}-2^{-1.5} \cos \left(2 t+\frac{\pi}{4}\right)}{1+\cos ^{2}(t)}+$ $\frac{0.25}{\sqrt{t} \Gamma(0.5)\left(1+\cos ^{2}(t)\right)}=\frac{-0.125-\frac{0.75}{\sqrt{t}(0.5)}-2^{-1.5} \cos \left(2 t+\frac{\pi}{4}\right)}{1+\cos ^{2}(t)}$ is changeable (see Figure 3), we are not able to use the above results and to make a conclusion.

Case 2. Consider the Lyapunov function $V_{1}(t, S, I)=\left(1+\cos ^{2}(t)\right) I^{2}$.

Case 2.1. The Caputo derivative of the Lyapunov function $V_{1}(t, S(t), I(t))$ is difficult to obtain so we will not use it.

Case 2.2. Dini fractional derivative. Using $1+\cos ^{2}(t)=1.5+0.5 \cos (2 t)$ and ${ }_{0}^{R L} D^{0.5} \cos (b t)=$ $\left.b^{0.5} \cos (b t+0.25 \pi)[38]\right)$, we obtain ${ }_{0}^{R L} D^{0.5}\left(1+\cos ^{2}(t)\right)={ }_{0}^{R L} D^{0.5}(1.5+0.5 \cos (2 t))=\frac{1.5}{t^{0.5} \Gamma(0.5)}+$ $\frac{1}{\sqrt{2}} \cos (2 t+0.25 \pi)$.

From (7), Example 2, Case 3, we obtain for the Dini fractional derivative

$$
\begin{aligned}
& \mathcal{D}_{(29)}^{+} V(t, S, I ; 0) \leq 2(-\mu(t)-\gamma(t)+\lambda(t)) I^{2}+I^{2}{ }_{0}^{R L} D^{0.5}\left(1+\cos ^{2}(t)\right) \\
& =I^{2}\left(2 \frac{-0.125-\frac{0.75}{\sqrt{t} \Gamma(0.5)}-2^{-1.5} \cos \left(2 t+\frac{\pi}{4}\right)}{1+\cos ^{2}(t)}\right. \\
& \left.+\frac{1.5}{t^{0.5} \Gamma(0.5)\left(1+\cos ^{2}(t)\right)}+\frac{1}{\left(1+\cos ^{2}(t)\right) \sqrt{2}} \cos (2 t+0.25 \pi)\right)\left(1+\cos ^{2}(t)\right) \\
& =-0.25 I^{2} .
\end{aligned}
$$

According to Theorem 1 and Remark 5, the FrDE (27) is stable w.r.t. the variable $I$.

Author Contributions: All authors contributed equally to the writing of this paper. All three authors read and approved the final manuscript.

Funding: Research was partially supported by the Fund NPD, Plovdiv University, No. MU17-FMI-007.

Conflicts of Interest: The authors declare that they have no competing interests.

\section{References}

1. Bagley, R.L.; Calico, R.A. Fractional order state equations for the control of viscoelasticallydamped structures. J. Guid. Contr. Dyn. 1991, 19, 304-311. [CrossRef]

2. Laskin, N. Fractional market dynamics. Phys. A Stat. Mech. Appl. 2000, 287, 482-492. [CrossRef]

3. Tavazoei, M.S.; Haeri, M. A note on the stability of fractional order systems. Math. Comp. Simul. 2009, 79, 1566-1576. [CrossRef]

4. Zaslavsky, G.M.; Stanislavsky, A.A.; Edelman, M. Chaotic and pseudochaotic attractors of perturbed fractional oscillator. Chaos 2006, 16, 013102. [CrossRef] [PubMed]

5. Ahmed, E.; El-Sayed, A.M.A.; El-Saka, H.A.A. Equilibrium points, stability and numerical solutions of fractional-order predator-prey and rabies models. J. Math. Anal. Appl. 2007, 325, 542-553. [CrossRef]

6. Li, C.P.; Deng, W.H.; Xu, D. Chaos synchronization of the Chua system with a fractional order. Phys. A 2006, 360, 171-185. [CrossRef]

7. Hilfer, R. Applications of Fractional Calculus in Physics; World Scientific: Singapore, 2000.

8. Machado, J.A.T.; Silva, M.F.; Barbosa, R.S.; Jesus, I.S.; Reis, C.M.; Marcos, M.G.; Galhano, A.F. Some Applications of Fractional Calculus in Engineering. Math. Probl. Eng. 2010, 2010. [CrossRef]

9. Fallahgoul, H.; Focardi, S.; Fabozzi, F. Fractional Calculus and Fractional Processes with Applications to Financial Economics; Academic Press: New York, NY, USA, 2016. 
10. Diethelm, K.; Ford, N.J. Analysis of fractional differential equations. J. Math. Anal. Appl. 2002, 265, 229-248. [CrossRef]

11. Daftardar-Gejji, V.; Jafari, H. Analysis of a system of nonautonomous fractional differential equations involving Caputo derivatives. J. Math. Anal. Appl. 2007, 328, 1026-1033. [CrossRef]

12. Momani, S.; Hadid, S. Lyapunov stability solutions of fractional integrodifferential equations. Int. J. Math. Math. Sci. 2004, 47, 2503-2507. [CrossRef]

13. Li, Y.; Chen, Y.; Podlubny, I. Stability of fractional-order nonlinear dynamic systems: Lyapunov direct method and generalized Mittag-Leffler stability. Comput. Math. Appl. 2010, 59, 1810-1821. [CrossRef]

14. Deng, W.H. Smoothness and stability of the solutions for nonlinear fractional differential equations. Nonlinear Anal. 2010, 72, 1768-1777. [CrossRef]

15. Li, C.P.; Zhang, F.R. A survey on the stability of fractional differential equations. Eur. Phys. J. Spec. Top. 2011, 193, 27-47. [CrossRef]

16. Agarwal, R.; O’Regan, D.; Hristova, S. Stability of Caputo fractional differential equations by Lyapunov functions. Appl. Math. 2015, 60, 653-676. [CrossRef]

17. Agarwal, R.; O'Regan, D.; Hristova, S. Stability by Lyapunov like functions of nonlinear differential equations with non-instantaneous impulses. J. Appl. Math. Comput. 2017, 53, 147-168. [CrossRef]

18. Henderson, J.; Hristova, S. Eventual Practical Stability and Cone Valued Lyapunov Functions for Differential Equations with "Maxima". Int. Commun. Appl. Math. 2010, 14, 515-523.

19. Hristova, S. Stability on a cone in terms of two measures for impulsive differential equations with "supremum". Appl. Math. Lett. 2010, 23, 508-511. [CrossRef]

20. Trigeassou, J.C.; Maamri, N.; Sabatier, J.; Oustaloup, A.; Lyapunov, A. Approach to the stability of fractional differential equations. Signal Process. 2011, 91, 437-445. [CrossRef]

21. Aguila-Camacho, N.; Duarte-Mermoud, M.A.; Gallegos, J.A. Lyapunov functions for fractional order systems. Commun. Nonlinear Sci. Numer. Simul. 2014, 19, 2951-2957. [CrossRef]

22. Duarte-Mermoud, M.A.; Aguila-Camacho, N.; Gallegos, J.A.; Castro-Linares, R. Using general quadratic Lyapunov functions to prove Lyapunov uniform stability for fractional order systems. Commun. Nonlinear Sci. Numer. Simul. 2015, 22, 650-659. [CrossRef]

23. Hu, J.B.; Lu, G.P.; Zhang, S.B.; Zhao, L.-D. Lyapunov stability theorem about fractional system without and with delay. Commun. Nonlinear Sci. Numer. Simul. 2015, 20, 905-913. [CrossRef]

24. Lakshmikantham, V.; Leela, S.; Devi, J.V. Theory of Fractional Dynamical Systems; Cambridge Scientific Publishers: Cambridge, UK, 2009.

25. Lakshmikantham, V.; Leela, S.; Sambandham, M. Lyapunov theory for fractional differential equations. Commun. Appl. Anal. 2008, 12,365-376.

26. Das, S. Functional Fractional Calculus; Springer: Berlin/Heidelberg, Germany, 2011.

27. Diethelm, K. The Analysis of Fractional Differential Equations; Springer: Berlin/Heidelberg, Germany, 2010.

28. Podlubny, I. Fractional Differential Equations; Academic Press: San Diego, CA, USA, 1999.

29. Samko, G.; Kilbas, A.A.; Marichev, O.I. Fractional Integrals and Derivatives: Theory and Applications; Gordon and Breach: New York, NY, USA, 1993.

30. Devi, J.V.; Rae, F.A.M.; Drici, Z. Variational Lyapunov method for fractional differential equations. Comput. Math. Appl. 2012, 64, 2982-2989. [CrossRef]

31. Baleanu, D.; Mustafa, O.G. On the global existence of solutions to a class of fractional differential equations. Comput. Math. Appl. 2010, 59, 1835-1841. [CrossRef]

32. Agarwal, R.; O’Regan, D.; Hristova, S.; Cicek, M. Practical stability with respect to initial time difference for Caputo fractional differential equations. Commun. Nonlinear Sci. Numer. Simul. 2017, 42, 106-120. [CrossRef]

33. Sadati, S.J.; Ghaderi, R.; Ranjbar, A. Some fractional comparison results and stability theorem for fractional time delay systems. Rom. Rep. Phys. 2013, 21, 94-102.

34. Stamova, I.; Stamov, G. Stability analysis of impulsive functional systems of fractional order. Commun. Nonl. Sci. Numer. Simul. 2014, 19, 702-709. [CrossRef]

35. Zhang, F.; Li, C.; Chen, Y. Asymptotical Stability of Nonlinear Fractional Differential System with Caputo Derivative. Int. J. Differ. Equ. 2011, 2011. [CrossRef]

36. Makhlouf, A.B. Stability with respect to part of the variables of nonlinear Caputo fractional differential equations. Math. Commun. 2018, 23, 119-126. 
37. Diethelm, K. A fractional calculus based model for the simulation of an outbreak of dengue fever. Nonlinear Dyn. 2013, 71, 613-619. [CrossRef]

38. Guce, I.K. On Fractional Derivatives: The Non-integer Order of the Derivative. Int. J. Sci. Eng. Res. $2013,4,1$. 